\title{
Ueber das Pancreassecrel;
}

\section{von Prof. Dr. C. Schmidt in Dorpat.}

Einige mifslungene Versuche, den Ausfülurungsgang der Bauchspeicheldriise dauernd zur Verwachsung mit der nächstliegenden durchbohrten Bauchwand zu bringen, den Saft nach aufsen abzuleiten, und so möglichst sichere Anhaltspunkte für die Betheiligung desselben an Verdaunngsprocefs zu gewinnen, hatten uns vor einigen Jahren zur Beschränkung auf temporäre Fisteln genöthigt *). Wir waren $\mathrm{zu}$ dem Schlusse gedangt, dafs derselbe theils zur Umwandlung des Stärkmehls der Nahrungsmittel in Gummi und Zucker bestimmt sey, theils durch seine Betheiligung am intermediären Darmkreislauf neben den andern Darmdrüsensecreten die rasche Bewegung der Flüssigkeiten innerhalb des Körpers mit vermittele, dafs er aber zur Verdauung der Albuminate oder Fette nicht mitwirke, deren erstere durch den Magensaft, letztere durch die Galle bewerkstelligt wird. Kürzlich ist es Hrn. Dr. Weinmann in Zürich unter L u dwig's Leitung geglückt, dauernde Pancreasfistcln bei Hunden berzustellen und gröfsere Quantiäten des Secretes aufzufangen ${ }^{* *}$ ). Die merkwürdigen Unterschiede zwischen der so erhaltenen wasserklaren leicht beweglichen Flüssigkeit und dem von uns unmiltelbar nach der Operation aus dem Pancreasgange mittelst Einführung silberner Canïlen tropfenweise aufgesammelten öldicken eiweilsähnlichen Fluidum hinsichtlich ihrer Concentration, wie ihrer absoluten Menge, liefsen uns dic Untersuchung wieder aufnelımen. Unsere jetzigen Erfahrungen ***) bestätigen sowohl die

4) P. Bidder u. C. Sclumidt, die Verdaunngssäfte etc. S. 240 bis 259.

**) Henle u. Pfeuffer's Zeitschr. f. rationelle lledicin, 1853, Bd. III, Heft 2.

*:s) Wir vereinigten uns dazu mit unserem jüngeren Freunde und Mitarbeiter Dr. K röger, dessen Inauguraldissertation : „S. K röger, de suceo pancreatico. Dorpati 1854" das Detail der Beobachtungsweisen enthält, deren Mittheilung hier zu weit führen würde. 
unserer Züricher Freunde, als unsere früheren eigenen, so weit die Fluida in gleicher Weise aufgefangen wurden.

Das Pancreassecret, nach der Operation aufgefangen, enthält 10 bis $11 \mathrm{pC}$. fester Stoffe, wovon 9 bis $10 \mathrm{pC}$. organische (Pancreasdiastase), 0,8 bis $0,9 \mathrm{pC}$. unorganische Basen und Salze; dasselbe aus der permanenten Fistel gewonnen enthält 1,5 bis $3,6 \mathrm{pC}$. fester Stoffe, wovon 1,5 bis $2,3 \mathrm{pC}$. organische (Pancreasdiastase etc.), 0,62 bis 0,75 pC. unorganische Basen und Salze; 1 Kilogrm. Thierkörper liefert von jenem 0,1 bis $0,2 \mathrm{Grm}$, von diesem 3 bis $5 \mathrm{Grm}$. binnen einer Stunde. Hinsichtlich der Analyse jenes können wir füglich auf unsere früheren Mittheilungen verweisen, deren Resultat wir schliefslich mit unseren neueren Erfahrungen parallelisiren wollen.

Das Pancreassecret, aus einer wohlverheilten permanenten Fistel gewonnen, ist ein klares, farbloses Fluidum von stark alkalischer Reaction, fade laugenhaftem Geschmacke, ohne besonderen Geruch. Seine Dichtigkeit ist 1,010 bis 1,011; es schäunt stark beim Schütleln, wandelt Stärkmehlkleister bei $37^{\circ} \mathrm{C}$. augenblicklich in Gummi und Zucker um, zerlegt die Fette, trübt sich bei $70^{\circ} \mathrm{C}$. und gerinnt vollständig bei $72^{\circ} \mathrm{C}$. in weifsen Flocken. Es wird durch Alkohol und Holzgeist coagulirt; das Coagulum (Pancreasdiastase) ist in reinem Wasser wieder klar löslich und zersetzt Stärkmehl und Butter gleich dem ursprünglichen. Durch Sieden wird diese Fermentwirkung aufgehoben, eben so durch Schwefelsäure, Chlorwasserstoffsäure, Salpetersäure, Metaphosphorsäure und Quecksilberchlorid, die starke weifse Niederschläge bilden, während Essigsäure, schweflige Säure, dreibasische Phosphorsäure, Kali und Ammoniak schon zu wenigen Tropfen hinzugesetzt die Winkung hindern, ohne Trübung oder Niederschlag zu bewirken. Die beiden letzteren, so wie kohlensaure Alkalien in gröfserer Menge zugeselzt, hindern das Gerinnen 
durch Hitze. Eisenchlorid giebt einen hellbraunen, Kupfersalze einen hellblauen flockigen Niederschlag, Jod und hydriodige Säure dicke rostfarbige, Chlor und Brom gelbe Niederschläge; Flüssigkeit und Niederschlag hahen die Fermentwirkung verloren. Strychnin-, Morphium- und Cinchoninsalze dagegen, Salicin, Harnstoff, Amygdalin, Aether, Cyanwasserstoffsäure, Galle und reines krystallisirtes glycocholsaures Natron beeinträchtigen dieselbe nicht im mindesten. Neutrales essigsaures Bleioxyd giebt einen dicken weifsen flockigen Niederschlag, im Ueberschusse des Fällungsmittels theilweise löslich; sowohl der Niederschlag, wie die Lösung wirken auf Stärkmehl. Magensaft verändert das Pancreassecret nicht, die Einwirkung auf Stärkmehl bleibt ungehindert. Traubenzuckerlösung mit demselben über Quecksilber abgesperrt, bleibt unverändert; nach drei Wochen beginnt Kohlensäureentwickelung, die einige Tage fortdauert, dann aufhört; die vorher alkalische Reaction des Gemisches ist in eine saure iibergegangen, der Geruch beim Oeffnen des Apparats nach zwei Monaten nicht unangenehm, keine Spur von Fäulnifs oder Buttersäurebildung. Amygdalin, zwei Monate mit Pancreassecret hingestellt, bleibt unzersetzt; Harnstoff bleibt anfangs unverändert, wird aber nach zwei bis drei Wochen allmälig in kohlensaures Ammoniak verwandelt.

Unter $0^{0}$ scheiden sich vor dem Erstarren durchsichtige, Quittenschleim ähnliche Gallertgerinnsel aus, die im Vergleich zu der übrig gebliebenen Flüssigkeit schwächer alkalisch sind und die Wirkung auf Stärkmehl im höheren Grade als letztere zeigen. Auf Glasplatten oder flachen Schalen in dünnen Schichten über Schwefelsäure ins Vacuum gebracht, trocknet das Secret zu farblosen durchscheinenden, Mundleim ähnlichen Massen ein, die in Wasser wieder aufschwellen und sich klar lösen, die Wirkung auf Stärkmehl bleibt dabei fast unverändert. Findet das Eintrocknen in dickeren Schichten 
und bei 30 bis $50^{\circ} \mathrm{C}$. statt, so wird ein bedeutender Theil zersetzt, und die Fermentwirkung ist dengemäls verringert.

1 Kilogrm. Thierkörper (Hund) liefert binnen einer Stunde :

๘. bei einem absoluten Körpergewicht von 8 Kilogrm. 5,03 Grm. à $2,16 \mathrm{pC}$. $=0,106 \mathrm{Grm}$. fester Substanz.

b. bei einem absoluten Körpergewicht von 18 Kilogrm. 3,11 Grm. à $1,99 \mathrm{pC} .=0,061 \mathrm{Grm}$. fester Substanz.

c. bei einem absoluten Körpergewicht von 26 Kilogrm. 2,99

Grm. à $2,45 \mathrm{pC}$. $=0,073 \mathrm{Grm}$. fester Substanz.

Jede der Beobachtungsreihen umfalst zahlreiche Einzelbestimmungen, so dafs die aufgeführten Mittel als möglichst wahrscheinliche Werthe zu betrachten sind. Für dieselbe Thiergattung ist demnach die relative Secretionsgrölse, wie wir es bereits bei der Gallensecretion beobachtet, direct proportional dem relativen Nahrungs - oder, mit andern Worten, Athmungs - oder Wärmebedürfnifs; je grölser das Thier, desto geringer verhältnilsmäfsig der durch Athmung und Nahrungszufuhr wieder zu ersetzende Wärmeverlust und demgemäls die Quantität zu liefernden Darmdrüsensecretes.

Im Mittel von sechs Bestimmungen wandelt $1 \mathrm{Grm}$. frisches Pancreassecret, worin 0,021 Grm. wasserfreie Substanz und zwar 0,014 Grm. organische Substanz (Pancreasferment), 0,007 Grm. unorganische Basen und Salze, bei $37^{\circ} \mathrm{C}$. eine halbe Stunde lang mit überschüssigem Stärkmehlkleisłer digerirt, 4,672 Grm. wasserfreies Stärkmehl um, so dafs je $1 \mathrm{Grm}$. trockener Pancreasdiastase 333,7 Grm. des lelzteren unter den erwähnten Verhältnissen darmaufsaugungsfähig zu machen vermag.

$\mathrm{Zu}$ den nachstehenden Analysen wurden drei zu verschiedenen Zeiten aufgefangene Portionen von 50 bis 100 Grm. verwendet; die Methode ergiebt sich theils aus den analytischen Daten, theils aus unseren früheren, a. a. 0 . mitgetheilten Untersuchungen. 
A. Klares, farbloses Secret; Dichtigkeit bei $15^{\circ} \mathrm{C}$. im Vacuo $=1,0106$.

42,426 Grm. frisches Secret hinterlassen eingetrocknet $0,985 \mathrm{Grm}$. bei $110^{\circ}$ trockenen Rïckstand, dieser geglüht $0,387 \mathrm{Grm}$. Kohle und kohlensäurehallige, geschmolzene, stark alkalische Asche, woraus

0,0017 Grm. Kohle, $0,292 "$ Chlorsilber, $0,00194 "$ Phosphorsäure $\quad$ +Kohle und Kohlensäure, den $0.00080 \%$ Kalk in Wasser unlöslichen Theil der $0,00036, \quad$ Magnesia mit Spuren $\begin{aligned} & \text { Asche bildend, aus der Analyso } \\ & \text { gröfserer Portionen ungewoge- }\end{aligned}$ gror Pancreassecretinengen borechnet.

$0,430 \mathrm{Grm}$. Chlorkalium + Chlornatrium, woraus 0,140 "Kaliumplatinchlorid; also 0,3872 Chlornatrium $\{0,0428$ Chlorkalium.

B. Physikalische Eigenschaften die vorigen.

83,107 Grm. frisches Secret hinterlassen eingetrocknet $1,668 \mathrm{Grm}$. bei $110^{\circ}$ trockenen Rückstand; dieser geglüht $0,7477 \mathrm{Grm}$. Kohle und kohlensäurehaltige Asche, woraus

0,0077 Grm. Kohle,
$0,880 \%$ Chlorsilber,
$0,00414 \%$ Phosphorsäure
$0,0045, \quad$ Kalk

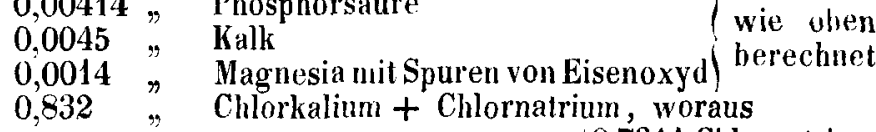
0,288 \%aliumplatinchlorid; also $\begin{aligned} & \text { 10,7311 Chlornatrium } \\ & 0,0943 \text { Chlorkalium. }\end{aligned}$

C. Physikalische Eigenschaften wie früher.

$80,556 \mathrm{Grm}$. frisches Secret hinterlassen eingetrocknet $1,267 \mathrm{Grm}$. bei $110^{\circ}$ trockenen Rückstand, dieser geglüht $0,691 \mathrm{Grm}$. Kohle und kohlensäurehaltige Asche, woraus

0,0105 Grm. Kohle,
0,544
Chlorsilber',
$0,00219 "$
0,00225 ,
Phosphorsäure
0,0007 ?
Kalk
0,740 "
Magnesia
wie oben berechnet
0,199 "
Chlorkalium + Chlornatrium, woraus
Kaliumplatinchlorid; also $\left\{\begin{array}{l}0,6792 \text { Chlornatrium } \\ 0,0608 \text { Chlorkalium. }\end{array}\right.$ 
Demnach enthalten 1000 Grm. Pancreassecret :

\begin{tabular}{|c|c|c|c|c|c|c|}
\hline \multicolumn{5}{|c|}{ I. Aus permanenter Fistel aufgefangen : } & \multicolumn{2}{|c|}{$\begin{array}{l}\text { II. Unmittelbar } \\
\text { nach der Opera- } \\
\text { tion aus tempn- } \\
\text { rärer Fistel er- } \\
\text { halten }{ }^{*} \text { ): }\end{array}$} \\
\hline & $\bar{A}$ & $\bar{B}$ & $\overline{\mathrm{C}}$ & Mittel\| & $\bar{A}$ & $\overline{\mathbf{B}}$ \\
\hline $\begin{array}{l}\text { Wasser } \\
\text { Feste Stoffe } \\
\end{array}$ & $\begin{aligned} 976,78 \\
23,22\end{aligned}$ & $\begin{array}{r}979,93 \\
20,07\end{array}$ & $\begin{array}{r}984,63 \\
15,37\end{array}$ & $\left|\begin{array}{r}980,45 \\
19,55\end{array}\right|$ & $\begin{array}{r}900,76 \\
99,24\end{array}$ & $\begin{array}{l}884,4 \\
115,6\end{array}$ \\
\hline $\begin{array}{l}\text { Organ. Sulsstanz (Ferment) } \\
\text { Unorgan. Basen u. Salze }\end{array}$ & $\begin{array}{r}16,38 \\
6,83\end{array}$ & $\begin{array}{r}12,45 \\
7,52\end{array}$ & $\begin{array}{l}9,21 \\
6,16\end{array}$ & $\begin{array}{r}12,71 \\
6,84\end{array}$ & $\begin{array}{r}90,44 \\
8,80\end{array}$ & - \\
\hline Natron (an das Ferment & & & & & & \\
\hline $\begin{array}{c}\text { gebunden) } \\
\text { Chlornatrium } \\
.\end{array}$ & $\begin{array}{l}3,818 \\
1,917\end{array}$ & $\begin{array}{l}2,858 \\
3,484\end{array}$ & $\begin{array}{l}3,249 \\
2,110\end{array}$ & $\begin{array}{l}3,31 \\
2,50\end{array}$ & $\begin{array}{l}0,58 \\
7,35\end{array}$ & - \\
\hline Chlorkalium . & 1,008 & 1,059 & 0,738 & 0,93 & 0,02 & - \\
\hline $\begin{array}{l}\text { Phösphors. Kalk : } \\
\text { Phosphors. Magnesia mit }\end{array}$ & 0,051 & 0,100 & 0,051 & 0,07 & 0,41 & - \\
\hline $\begin{array}{l}\text { Spuren von Eisenoxyd } \\
\text { Phosphors. Natron (drei- }\end{array}$ & 0,024 & 0,006 & 0,005 & 0,01 & 0,12 & - \\
\hline $\begin{array}{l}\text { basisch) } \\
\text { Kalk (an das Furment ge- }\end{array}$ & 0,015 & - & - & 0,01 & - & - \\
\hline bunden) $\cdot$. . . & - & - & - & - & 0,32 & - \\
\hline Magnesia (desgl.) . . & - & 0,015 & 0,006 & 0,01 & - & - \\
\hline Dichtigkeit . . . & $\begin{array}{l}6,833 \\
-\end{array}$ & $\begin{array}{l}7,522 \\
1,0106\end{array}$ & $\begin{array}{c}6,159 \\
-\end{array}$ & 6,84 & $\begin{array}{l}8,80 \\
1,0306\end{array}$ & $\overline{-}$ \\
\hline
\end{tabular}

Diese constanten bedeutenden Unterschiede können nicht durch blofse Wasseraufsaugung in Folge des operativen Eingriffs, oder gar Wasserverdunstung während des Aufsammelns erklärt werden. Eine solche hätte den Gehalt an organischen und unorganischen Stoffen gleichzeitig entsprechend gesteigert, während ersterer letzteren unverhälınifsmälsig uberwiegt; aus dem Mittel der Versuchsreihen I. abgeleitet hätte auf 8,80 Grm. unorganischer Basen und Salze 16,35 Grm. Pancreasferment etc. statt 90,44 erhalten werden müssen. Legen wir endlich auch auf die Verschiedenheit ersterer bei der geringen Menge für die Analysen II. verwendbaren Materials kein übertriebenes Gewicht, so kann

*) a. a. 0. S. 245 . 
doch die bedeutende Verringerung des Gehalts an scliwach gebundenem Alkali (NaO) gegenüber den Chloriden $(\mathrm{NaCl}$ und $\mathrm{KCl}$ ) keinem Versuchsfehler zugeschrieben werden, da durch letztere (zu heftiges Glühen der kohlehaltigen Asche bei Luftzutritt z. B.) der Chlorgehalt durch Verflüchligung verringert, nicht aber, wie im vorliegenden Falle, gesteigert werden muls; bei $0,142 \mathrm{Grm} . \mathrm{AgCl}$, die zur Chlorbestimmung II. dienten, können endlich Wägungsfehler u. dergl. höchstens $0,1 \mathrm{pC}$. des Chlornatriumgehalts zweifelhaft machen. Wir sind demnach hier vorläufig zur Annahme einer älınlichen Rückwirkung der Innervation auf den Secretionsprocefs genöthigt, wie sie Ludwig für die Speicheldrüsen, Bernard für die Nieren nach Reizung der Drüsenkanälchen $(\alpha)$ oder des Vagusursprunges im Gehirn aufser Zweifel gesetzl; wir sagen „vorläufig“, da von der blofsen Thatsache bis zu rationeller Erklärung noch ein weiles Gebiet exacter Forschung vorliegt.

Hinsichtlich aller übrigen Beziehungen des pancreatischen Saftes, namentlich seiner Nichtbetheiligung bei der Fett- und Albuminatverdauung, haben wir unseren früheren experimentellen Beweisen nichts hinzuzufügen, deren Gültigkeit durch die neuere Erweiterung unserer Kenntnisse über die normale mittlere quantitative Zusammensetzung dieses Secrets nicht im Mindesten beeinträchtigt wird. Nur sein Verhältnifs zum intermediären Darmkreislauf wird dadurch wesentlich verändert; es gestaltet sich nach Anlage permanenter Fisteln bei derselben Thiergaltung (Hund) mit Beziehung auf das Leber - und Magendrüsensecrel folgendermalsen : 
1 Kilogrm. Thierkörper (Hund) secernirt binnen 24 Stunden :

\begin{tabular}{|c|c|c|c|c|c|c|c|c|}
\hline & & & & Wasser & $\begin{array}{c}\text { Organ. } \\
\text { Stoffe }\end{array}$ & $\begin{array}{l}\text { Unorgan. } \\
\text { Stoffe }\end{array}$ & $\begin{array}{c}\text { Chlorwasser- } \\
\text { stoff }\end{array}$ & Natron \\
\hline \multicolumn{4}{|c|}{$\begin{array}{l}\text { speichelhaltigen Magensaft } \\
100 \mathrm{Grm} \text {., worin }\end{array}$} & 97,12 & 1,75 & 1,13 & 0,270 & - \\
\hline \multirow{2}{*}{$\begin{array}{l}\text { Falle } \\
\text { Pancreas- } \\
\text { secret }\end{array}$} & & $"$ & $"$ & 19,01 & 0,99 & 0,10 & - & 0,059 \\
\hline & 89 & $"$ & $"$ & 87,24 & 1,15 & 0,61 & - & 0,293 \\
\hline Sunme & 209 & Grm & worin & 203,37 & 3,89 & 1,84 & äq. 0,270 NaO & 0,352 \\
\hline
\end{tabular}

Die Gesammlmenge des circulirenden Blutes $=200 \mathrm{Grm}$. gesetzt, wovon $176 \mathrm{Grm}$. Wasser, 22,41 Grm. organische Stofle, 1,59 Grm. unorganische Bestandtheile, 0,89 Grm. Chlornatrium und Chlorkalium, so ergiebt sich, dafs dit: Hälfte des Wassers $\frac{2}{5}$ der unorganischen Salze binnen 24 Stunden intermediär durch die Pancreasdruse in dem Darmrohr secernirt und aus letzterem in den Kreislaul wieder aufgenommen werden, dafs über die Hälfte der vorhandenen Kochsalzmenge $(0,270 \mathrm{HCl}$ äq. $0,433 \mathrm{NaCl}, 0,293 \mathrm{NaO}$ äq. 0,553 $\mathrm{NaCl}$ ) sich täglich in Chlorwasserstoffsäure und Natron spaltet, von denen jene ( $\mathrm{HCl}$ ) durch die Magendrisen, dieses ( $\mathrm{NaO}$ ) vorzugsweise durchs Pancreas ausgeschieden werden, um im weitern Verlauf des Intestinaltractes vereint und als Chlornatrium wieder aufgesogen, den Kreislauf wieder zu beginnen.

Für den Menschen von 64 Kilogrm. Körpergewicht würde mit Zugrundelegung der geringsten, an dem Hunde $\mathrm{C}$ von 26 Kilogrm. Gewicht ermittelten flüssigen Secretionsmenge von $72 \mathrm{Grm}$. für 1 Kilogrm. Thierkörper, das Tagesquantum auf 4,6 Kilogrm. zu veranschlagen seyn.

Ein erwachsener Mann von 64 Kilogrm. Körpergewicht exspirirt binnen 24 Stunden, durchschnittlich 786 Grm. Kohlensäure und secernirt $32 \mathrm{Grm}$. Harnstoff, äquivalent 
92,70 Grm.Albuminaten $=42,73 \mathrm{Grm}$. Respirationskohlenstof *) 786 Grm. Kohlensäure $=214,36$, Kohle

Differenz $=171,63 \%$ Kohle, äq. 386,17 Grm. Stärkmehl, zu deren Umwandlung in Zucker, d. h. Ueberführung in resorptionsfähige Form, $82,66 \mathrm{Gm}$. pancreatischen Saftes genügen.

Von den binnen 24 Stunden secernirten 4,6 Kilogrm. pancreatischen Saftes genügen daher den eigentlichen Verdaunngsfunctionen 0,08 Kilogrm., der Rest $=4,52$ Kilogrm., d. h. 98,2 pC. der ausgeschiedenen und in den Darm ergossenen Menge ist für letzlere entbehrlich. Erwägt man, dafs reine Fleischfresser mitunter relativ grófsere Bauchspeicheldrüsen besitzen, als Herbivoren gleicher Ordnungen, dafs ferner die Fett- und Albuminalverdaung bei Ausschlufs des Pancreassecretes eben so vollständig erfolgt, als bei Gegenwart desselben**), so wird der Schlufs hinlänglich berechtigt erscheinen, dals dasselbe vorzugsweise den intermediären Wasserkreislauf zu vermitteln, und das, nach Abspaltung der, Chlorwasserstoffsäure des Kochsalzes, im weiterströmenden Blute angehäufte schwach gebundene Natron sofort als stark alkalisches Natron-Albuminoïd (DiastaseNatron) aus jenem, Behufs Herstellung des typischen Gleichgewichts zwischen Säuren und Basen, zu eliminiren bestimmt, nur nebenher als eigentliches Verdaunngsagens, im engeren Sinne des Wortes, zu betrachten ist.

\footnotetext{
*) Vergl. Verdauungssäfte u. s. w. S. 303, Tab. IIl.

**) ibid. Artikel "frallea.
} 